\title{
Probing collective multi-electron dynamics in xenon with high-harmonic spectroscopy
}

\author{
A. D. Shiner ${ }^{1 \dagger}$, B. E. Schmidtt ${ }^{1, \dagger}$, C. Trallero-Herrero ${ }^{1,3}$, H. J. Wörner ${ }^{1,4}$, S. Patchkovskii ${ }^{1}$, P. B. Corkum ${ }^{1}$, \\ J-C. Kieffer ${ }^{2}$, F. Légaré ${ }^{2}$ and D. M. Villeneuve ${ }^{1 \star}$
}

\begin{abstract}
High-harmonic spectroscopy provides a unique insight into the electronic structure of atoms and molecules ${ }^{1-5}$. Although attosecond science holds the promise of accessing the timescale of electron-electron interactions, until now, their signature has not been seen in high-harmonic spectroscopy. We have recorded high-harmonic spectra of atoms to beyond $160 \mathrm{eV}$, using a new, almost ideal laser source with a wavelength of $1.8 \mu \mathrm{m}$ and a pulse duration of less than two optical cycles. We show that we can relate these spectra to differential photoionization cross-sections measured with synchrotron sources. In addition, we show that the highharmonic spectra contain features due to collective multielectron effects involving inner-shell electrons, in particular the giant resonance in xenon. We develop a new theoretical model based on the strong-field approximation and show that it is in agreement with the experimental observations.
\end{abstract}

Measuring and understanding the electronic structure and correlated dynamics of matter on its natural timescale represents the main thrust of ultrafast laser science. Electron correlations affect essential properties of complex systems ranging from configuration interactions in molecules to cooperative phenomena in solids, such as superconductivity. Our knowledge of the electronic structure of matter originates from several decades of research on photoionization and photoelectron spectroscopy ${ }^{6-8}$, mainly driven by the development of synchrotron-based sources. Recent advances in strong-field physics have opened an alternative approach to probing both the electronic structure ${ }^{1,9}$ and the dynamics ${ }^{10-12}$ of molecules using table-top laser sources. These new methods rely on the recollision of an electron, removed from the molecule by a strong laser field, with its parent ion $^{13}$, as illustrated in Fig. 1a. The electronic structure of the molecule is encoded in the emitted high-harmonic spectrum through the amplitude and phase of the photorecombination matrix elements ${ }^{4,11,14,15}$.

We use high-harmonic spectroscopy to investigate a new class of collective electronic dynamics - induced and probed by the recombining electron. The kinetic energy of the returning electron is usually much larger than the difference between electronic energy levels of the parent ion. Consequently, inelastic scattering followed by recombination is energetically possible, as illustrated in Fig. $1 \mathrm{~b}$. Using the xenon atom as an example, we demonstrate that such processes indeed occur and that they can locally enhance the efficiency of high-harmonic generation (HHG) by more than one order of magnitude. We show that such a seemingly complex pathway contributes significantly to the phase-matched process. This observation uncovers a new unexpected facet of high-harmonic spectroscopy-it provides access to electron correlations that are otherwise very difficult to observe. Our results indicate that electron-electron excitations may be ubiquitous in high-harmonic spectroscopy experiments. Highharmonic spectroscopy gives access to multi-electron dynamics through their spectral signature, much as in photoionization studies, but it offers the additional potential of attosecond temporal resolution. Until recently, HHG was interpreted within the single-active-electron approximation. HHG experiments in preformed, transition-metal plasmas ${ }^{16}$ demonstrated significant enhancements of a single harmonic order. These enhancements were recently interpreted ${ }^{17}$ to be caused by Fano autoionizing resonances and $3 d-3 p$ transitions, underlining the importance of multi-electron effects in HHG.

We used a new laser source that is almost ideal for spectroscopic studies ${ }^{18}$, having a wavelength of $1.8 \mu \mathrm{m}$ and a duration of less than 2 optical cycles. The source is described in the Methods section and in the Supplementary Information. With its long wavelength, it creates a recollision electron whose energy can exceed $100 \mathrm{eV}$, even for low-ionization-potential systems such as small organic molecules.

HHG spectra were recorded for the noble gases, argon, krypton and xenon, using a thin phase-matched gas jet described in the Methods section. Results are shown in Figs 2 and 3 and demonstrate the smooth spectra that are possible with this laser source. The limit of $160 \mathrm{eV}$ is imposed by the spectrometer. We now show how the photoionization cross-section (PICS) can be measured with these spectra. It has been shown that the three-step model for HHG (ref. 13) can be approximately factorized into three terms $s^{1,2,15,19}$ corresponding to ionization $(I)$, electron propagation $(W)$, and recombination $\left(\sigma^{\mathrm{r}}\right)$. Although the range of validity for this expression has not been fully explored, it is sufficiently accurate to allow us to compare the HHG spectra of Xe and $\mathrm{Kr}$, both of which involve ionization from $p$ orbitals.

$$
S\left(E_{\Omega}\right)=I(F, \omega) W(E) \sigma^{\mathrm{r}}(E)=\mathbb{W}(E) \sigma^{\mathrm{r}}(E)
$$

Here $S\left(E_{\Omega}\right)$ is the HHG power spectrum, $E_{\Omega}=E+I_{p}$ is the emitted photon energy, $E$ is the electron kinetic energy, $\omega$ is the laser frequency, $F$ is the laser field and $I_{p}$ is the ionization potential. The photorecombination cross-section $\sigma^{\mathrm{r}}$ is related to the photoionization cross-section $\sigma^{i}$ through the principle of detailed balance ${ }^{2,20}$.

To demonstrate that our laser source produces an almost flat recolliding electron wave packet, we plot the differential PICS for krypton $^{21}$ together with our measured HHG spectrum in Fig. 2. The good agreement tells us that $\mathbb{W}(E)$ is virtually flat. The development

\footnotetext{
${ }^{1}$ Joint Attosecond Science Laboratory, National Research Council of Canada and University of Ottawa, 100 Sussex Drive, Ottawa, Ontario K1A OR6, Canada, ${ }^{2}$ Institut National de la Recherche Scientifique, 1650 boul. Lionel-Boulet, Varennes, Québec J3X 1S2, Canada, ${ }^{3}$ J. R. Macdonald Lab, Physics Department, Kansas State University, Manhattan, Kansas 66506, USA, ${ }^{4}$ Labortorium für Physikalische Chemie, ETH Zürich, Wolfgang-Pauli-Strasse 10 , 8093 Zürich, Switzerland. ${ }^{\dagger} T$ These authors contributed equally to this work. ${ }^{\star}$ e-mail: david.villeneuve@nrc.ca.
} 


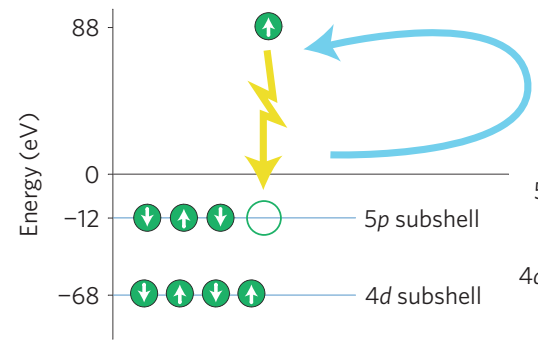

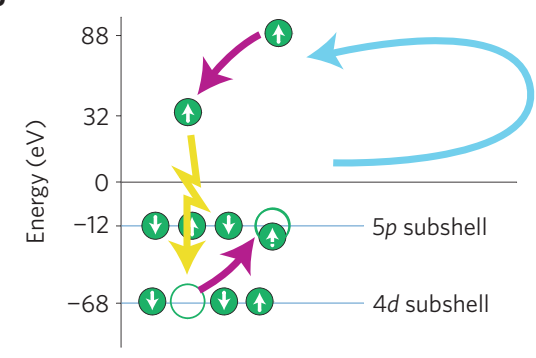

Legend:

lonization

Inelastic scattering

Recombination

Figure 1 | Steps for harmonic generation. a, In the usual three-step model, an electron is tunnel ionized from the valance shell, accelerates in the

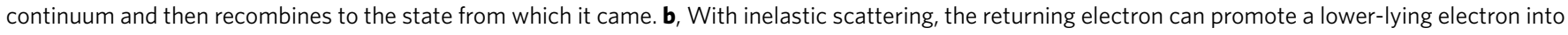
the valance band and then recombine to the vacancy in the lower-lying state. In both cases, a 100 eV photon is emitted by recombination to a $5 p$ vacancy (a) or a $4 d$ vacancy (b).

of this new source allows us to see many features through HHG that were previously not accessible with traditional laser sources. For example in the krypton spectrum (Fig. 2), we observe a Cooper minimum at $80 \mathrm{eV}$. Cooper minima have been reported in HHG experiments for argon ${ }^{4,22}$, but not for krypton. With our new $1.8 \mu \mathrm{m}$ source, we are able to report the first observation of the Cooper minimum in krypton in HHG.

We now use the measured Kr HHG spectrum and the PICS from the literature to accurately determine the recolliding electron wave-packet spectrum $\mathbb{W}(E)$ by setting $\mathbb{W}(E)=S_{\mathrm{Kr}}\left(E_{\Omega}\right) /{\sigma_{\mathrm{Kr}}}^{i}\left(E_{\Omega}\right)$, using a similar approach to that used in ref. 1 . We then divide the measured HHG spectrum for xenon with this term, to extract the photoionization cross-section for xenon: $\sigma_{\mathrm{Xe}}{ }^{i}=S_{\mathrm{Xe}}\left(E_{\Omega}\right) / \mathbb{W}(E)$. As this procedure divides one spectrum by another, experimental details such as grating reflectivity and detector response cancel out. In addition, the proportionality factor ${ }^{2,20}$ relating $\sigma^{i}$ and $\sigma^{\mathrm{r}}$ approximately cancels out when the ionization potentials are similar. The experimentally derived $\sigma_{\mathrm{Xe}}{ }^{i}$ is plotted as the blue line in Fig. 3, together with the PICS from synchrotron experiments. The excellent agreement shows that the HHG spectrum contains detailed information about the electronic structure of atoms, imprinted through the photorecombination cross-section. It is remarkable that the intense laser field can be neglected.

We now study the xenon results, shown in Fig. 3. The most striking feature is the pronounced peak around $100 \mathrm{eV}$. This peak has been extensively studied in the context of photoionization, and led to the development of a theory that included electron-electron correlations during photoionization ${ }^{23}$. The $100 \mathrm{eV}$ peak is interpreted as being due to the influence of $4 d$ electrons, which have a large photoionization cross-section in this region owing to a shape resonance. Energy-resolved measurements ${ }^{24}$ have shown that the photoionization cross-section of the $5 p$ shell is strongly enhanced around $100 \mathrm{eV}$ through electron-electron interactions with the $4 d$ subshell.

The first step in HHG is tunnel ionization from the most weakly bound electrons, the $5 p$ electrons in the case of xenon. The probability of tunnel ionizing a $4 d$ electron whose binding energy is $68 \mathrm{eV}$ is vanishingly small $\left(10^{-51}\right)$. So how is it that the photorecombination process in HHG can manifest the influence of the $4 d$ shell? Figure $1 \mathrm{~b}$ illustrates the Coulomb interaction between the returning continuum electron and a bound $4 d$ electron. An exchange of energy takes place, resulting in the promotion of a $4 d$ electron to fill the $5 p$ hole. This causes the continuum electron to lose $56 \mathrm{eV}$ (the difference between binding energies of the two subshells), and hence to have the correct kinetic energy to experience the quasibound continuum state that enhances the $4 d$ cross-section. The decelerated electron then recombines to the $4 d$

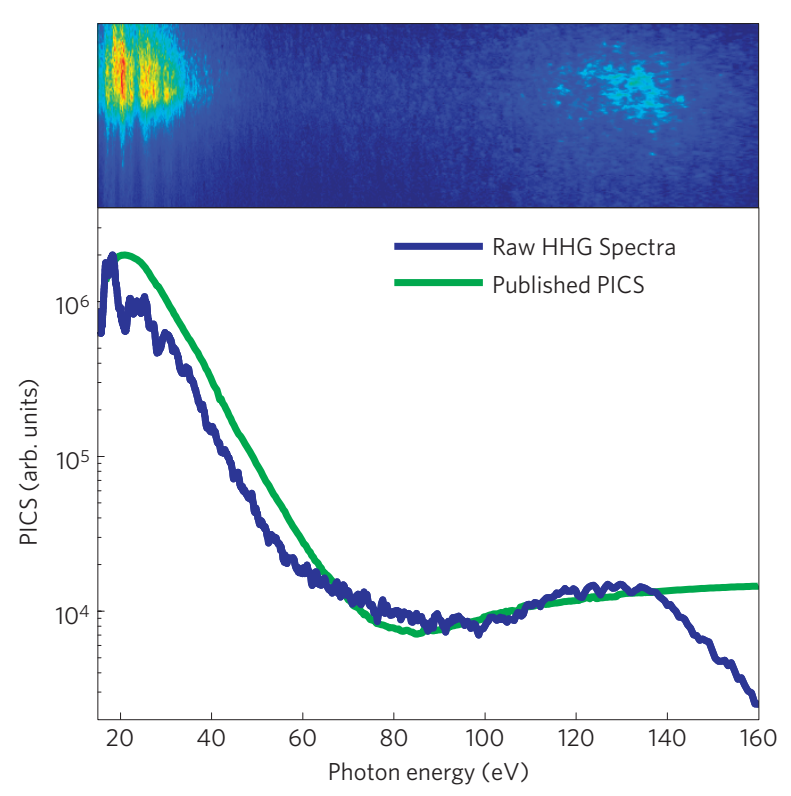

Figure 2 | Results for krypton. Top, raw HHG spectrum from krypton at $1.8 \times 10^{14} \mathrm{~W} \mathrm{~cm}^{-2}$ in an image taken from a CCD (charge-coupled device) camera. The horizontal scale has been stretched to be linear in frequency rather than wavelength. Bottom, experimental spectrum (blue) from integrating the CCD image vertically, including the Jacobian of the transformation from wavelength to frequency; published photoionization cross-section $^{21}$ (PICS; green).

hole and emits a photon whose energy is the same as that of the direct channel, owing to energy conservation.

The smooth green curve in Fig. 3 is derived from a complete multi-electron calculation ${ }^{25}$ that includes contributions from all subshells. We now describe a simplified model in which electron correlations are included in the recombination step of HHG. In the Supplementary Information, we show how this is included in a strong-field approximation model. Briefly, we include the Coulomb interaction between the returning electron that was ionized from the $5 p$ state and the other electrons of the ion. For clarity, we include only the $4 d$ state, although by summation other states can be easily included. Following ref. 23, the recombination dipole moment can be written as $D(\Omega)=d_{5 p}+d_{4 d} A_{c}$, where the single electron dipole moments are $d_{5 p}=\left\langle\phi_{5 p}|r| \chi_{5 p}\right\rangle$ and $d_{4 d}=\left\langle\phi_{4 d}|r| \chi_{4 d}\right\rangle$. The Coulomb interaction term is $A_{\mathrm{c}}=\left\langle\phi_{5 p} \chi_{4 d}\left|\left(\left|r_{1}-r_{2}\right|^{-1}\right)\right| \phi_{4 d} \chi_{5 p}\right\rangle / \Delta I_{p}$. Here the bound states (labelled by $\phi$ ) are wavefunctions in a Hartree-Fock 


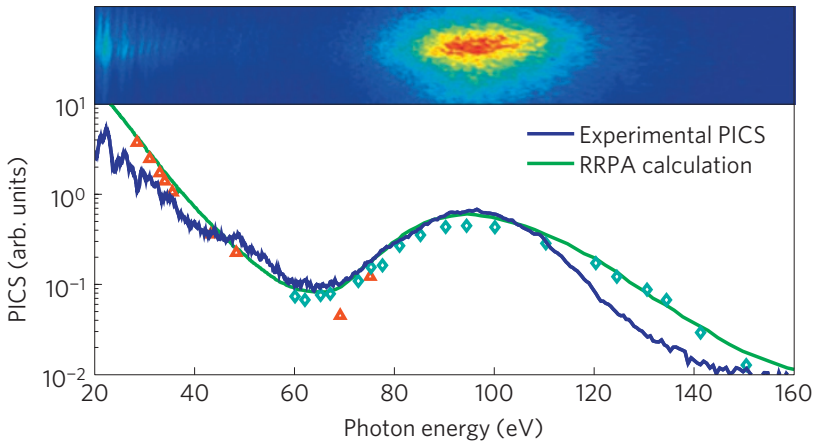

Figure 3 | Results for xenon. Top, the raw HHG spectrum from xenon at an intensity of $1.9 \times 10^{14} \mathrm{~W} \mathrm{~cm}^{-2}$. The horizontal scale has been stretched to be linear in frequency rather than wavelength. Bottom, experimental HHG spectrum divided by the krypton wave packet (blue) and the relativistic random-phase approximation (RRPA) calculation of the xenon photoionization cross-section (PICS) from ref. 25 (green). The red and green symbols are PICS measurements from refs 31 and 24 respectively, each weighted using the anisotropy parameter calculated in ref. 25.

model, and electron exchange is included through antisymmetrized products. The continuum states correlated with the hole states are labelled as $\chi_{5 p}$ and $\chi_{4 d}$. Each continuum state contains a full range of angular momentum components, only two of which contribute to the recombination.

Figure $4 \mathrm{a}$ shows the individual dipole moments for the $5 p$ and $4 d$ channels, as well as the Coulomb interaction term $A_{c}$. It can be seen that the direct $5 p$ channel alone cannot explain the observed peak at $100 \mathrm{eV}$. The total calculated cross-section including the electron-electron correlations is shown in Fig. 4 b, along with the experimentally recorded normalized HHG spectrum from Fig. 3. Our simplified model considers recombination with the $4 d(m=0)$ level, $\left(A_{\mathrm{c}}=0\right.$ for the other $m$ levels) and neglects further interactions between the other $4 d$ electrons. The model in ref. 25, green curve in Fig. 3, includes second-order interactions between the other $4 d$ electrons. We also note that the same calculation done for krypton $4 p / 3 d$ or for xenon $5 p / 5 s$ does not predict a giant resonance peak, in agreement with the experiment. It is the coincidence of two factors that gives rise to the $100 \mathrm{eV}$ peak in xenon-the strong electron-electron coupling just above the threshold of the $4 d$ level, and the strong transition dipole of the $4 d$ state at the same energy.

Although we have successfully predicted the observed HHG spectrum in xenon, it is not obvious that the $4 d$ channel will be phase matched, a necessity for HHG. Does the electron-electron interaction cause a loss of the coherence that is necessary for HHG phase matching? The generalized HHG model described in the Supplementary Information shows that coherence is indeed maintained. In the strong-field approximation, the ion evolves with a phase given by its total energy; the continuum electron gains a phase given by the classical action. After the collisional excitation, both the ion and the continuum electron exchange energy, but the total energy remains the same, meaning that the excitation time does not affect the emission phase, and coherence is maintained.

The observation of the $100 \mathrm{eV}$ peak in xenon in the HHG spectrum is striking in several ways. It reinforces the observation that the HHG spectrum is largely determined by the photoionization cross-section $^{2,4,15}$. In fact it has been predicted ${ }^{15}$ that the xenon giant resonance will appear in HHG spectra. The agreement between our measured spectrum and the previously measured photoionization cross-section is remarkable because it represents a clear observation of electron-electron correlations and excitation of the ion in HHG. Indeed, it is quite likely that collisional excitation followed by recombination to an inner-shell orbital is a general phenomenon in HHG whose experimental evidence has been directly observed

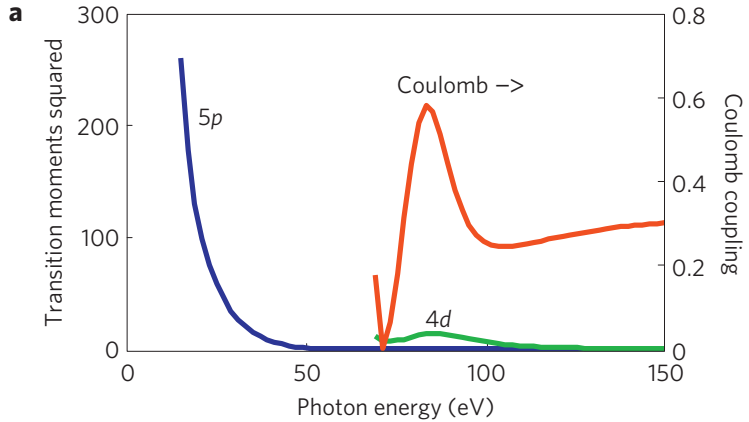

b

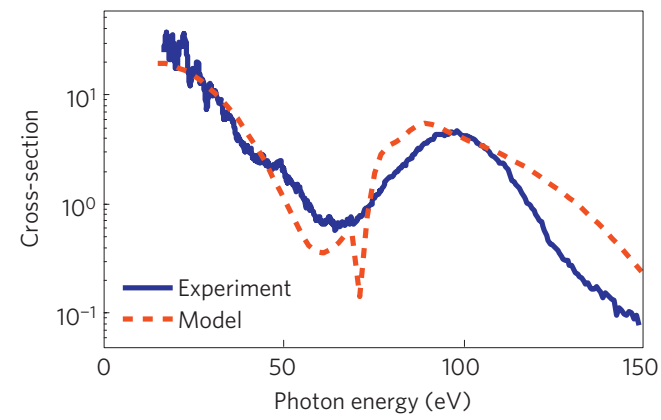

Figure 4 | Prediction of our theoretical model. a, The single-electron transition dipole moments (squared) for recombination to $5 p$ holes and $4 d$ holes in xenon. The electron-electron Coulomb interaction $\left|A_{c}\right|^{2}$

responsible for excitation of a $4 d$ electron to the $5 p$ hole is shown as a red curve (plotted against the right axis). b. The red dashed curve shows the predicted photorecombination cross-section, including both the direct $5 p$ channel and the indirect electron-electron interaction through the $4 d$ channel. The blue solid curve shows the experimentally obtained HHG cross-section of xenon from Fig. 3.

for the first time as a result of the newly developed sub-two-cycle infrared laser source.

Our results have three important implications. First, the high selectivity of tunnel ionization shows how photorecombination dynamics of the valence-shell orbital of molecules can be isolated and measured. In the photoionization of atoms, individual orbitals are easily identified through the kinetic energy of the photoelectrons. In molecules, however, photoelectron spectra of different orbitals overlap, inhibiting the selective observation of the PICSs over large energy intervals. Second, they show that the single-active-electron approximation can break down for HHG. Electron correlations beyond exchange interactions have been neglected until recently. We show that Coulomb correlations need to be included even at the qualitative level. Such interactions are ubiquitous in complex atoms and molecules. The giant resonance that we observe in high-harmonic spectroscopy for the first time is indeed known from photoionization experiments to persist from atoms into molecules and into the solid state. Third, owing to the factorization of HHG to explicitly include the field-free photorecombination cross-section, they demonstrate how the large body of spectroscopic data accumulated over the past decades will be useful in choosing optimal media for attosecond pulse generation in the quest to extend the photon energy ${ }^{26,27}$.

Our results also suggest new opportunities for future experiments. We have shown that HHG provides access to electronic structure information of inner shells through an unexpected mechanism. This opens the possibility to study electronic structure and correlations in inner shells of molecular systems where very little is known. Giant resonances as we have observed in xenon also offer interesting systems for attosecond time-resolved studies. Converting the $30 \mathrm{eV}$ measured width of the resonance into a lifetime, we 
obtain 23 attoseconds. Using an attosecond pulse centred at $100 \mathrm{eV}$ to photoionize xenon from the $4 d$ shell into the region of the giant resonance and subsequently streaking the photoelectron, should reveal the signature of the multi-electron dynamics. Such a measurement carried out in neon has recently revealed an unexpected temporal shift between the emission from different subshells ${ }^{28}$. Alternatively, the timescale of the electron correlations might be directly observable in high-harmonic experiments, if the electron is accelerated by the laser field after interaction, resulting in a phase shift of the emission; such an effect could be probed by varying the laser intensity or wavelength.

\section{Methods}

Source. Harmonic generation was driven by a $1.8 \mu \mathrm{m}, 11 \mathrm{fs}$ laser pulse. Details of this source have been published elsewhere ${ }^{18}$ and are also given in the Supplementary Information. In short, the $800 \mathrm{~nm}$ radiation from a Ti:sapphire laser (Thales, $100 \mathrm{~Hz}, 30 \mathrm{fs}$ ) was downshifted by an optical parametric amplifier (Light Conversion TOPAS-HE) producing approximately $1 \mathrm{~mJ}$ of radiation at $1.8 \mathrm{um}$ with a nominal pulse duration of $52 \mathrm{fs}$. This pulse was directed into a hollow-core fibre filled with 1.2 bar of argon gas, where its spectrum was broadened through self-phase modulation and self-steepening, and then compressed through anomalous dispersion in $2.0 \mathrm{~mm}$ of fused silica and a transmissive polarizer. This resulted in a nearly transform-limited $11 \mathrm{fs}$ pulse, which is only 1.9 optical cycles in duration. The spatial quality of the beam is excellent, owing to the spatial filtering properties of the fibre. The laser pulse is collimated after the hollow-core fibre, then focused into the vacuum chamber with a mirror with a focal length of $250 \mathrm{~mm}$. The focus was positioned before the gas jet, and optimized to favour phase-matched short trajectories and to suppress long trajectory harmonics.

Phase matching. Our intention is to apply high-harmonic spectroscopy to various rare-gas atoms. Unlike gas-source geometries that are designed to optimize production of extreme-ultraviolet emission, we employ a thin gas jet target in which collective effects are minimized. The gas sample is emitted from a pulsed valve with a $500 \mu \mathrm{m}$ orifice and a backing pressure of $3 \mathrm{bar}$. We have previously verified that the extreme-ultraviolet emission scales quadratically with gas pressure ${ }^{29}$, demonstrating that the target is operating in the phase-matched regime. The resulting high-harmonic spectrum is therefore a reflection of the single-atom response.

Spectrometer. Harmonic radiation produced in the gas jet passed into an extreme-ultraviolet spectrometer where the beam was spectrally dispersed by a 1,200 line $\mathrm{mm}^{-1}$ spherical holographic grating (Shimadzu 30-002). This type of grating has significantly lower second-order dispersion when compared with typical mechanically ruled gratings. The dispersed spectrum was imaged with a microchannel plate backed by a phosphor screen. The resulting spectrum was recorded with a high-dynamic-range digital camera.

Spectrometer calibration. The spectrometer was calibrated by putting oxygen into the pulsed jet and using several millijoules of circularly polarized $800 \mathrm{~nm}$ radiation while increasing the microchannel plate gain. We then recorded the plasma emission lines, using a method similar to that in ref. 30. By assigning the observed recombination lines to oxygen transitions known from the literature, we achieve a calibration of the spectrometer. Details of this procedure are given in the Supplementary Information.

Intensity calibration. The absolute laser intensity in the jet was calibrated by measuring the ion yield as a function of laser pulse energy. The intensity dependence of the mode-integrated ion yield for the target gas was then calculated using the Yudin-Ivanov non-adiabatic ionization model parameterized by the measured laser wavelength and pulse duration. The proportionality factor relating the pulse energy measured outside the chamber to the peak intensity in the interaction volume was found by fitting the measured ion yield to the intensity-dependent yield calculated from the model. We have published this procedure as part of our previous work ${ }^{29}$ and show details in the Supplementary Information.

Received 9 November 2010; accepted 31 January 2011; published online 6 March 2011; corrected online 7 March 2011

\section{References}

1. Itatani, J. et al. Tomographic imaging of molecular orbitals. Nature 432, 867-871 (2004).

2. Le, A-T., Lucchese, R. R., Tonzani, S., Morishita, T. \& Lin, C. D. Quantitative rescattering theory for high-order harmonic generation from molecules. Phys. Rev. A 80, 013401 (2009).

3. Mairesse, Y., Levesque, J., Dudovich, N., Corkum, P. B. \& Villeneuve, D. M. High harmonic generation from aligned molecules-amplitude and polarization. J. Mod. Optics 55, 2591-2602 (2008).

4. Wörner, H. J., Niikura, H., Bertrand, J. B., Corkum, P. B. \& Villeneuve, D. M. Observation of electronic structure minima in high-harmonic generation. Phys. Rev. Lett. 102, 103901 (2009).
5. Haessler, S. et al. Attosecond imaging of molecular electronic wavepackets. Nature Phys. 6, 200-206 (2010).

6. Berkowitz, J. Photoabsorption, Photoionization and Photoelectron Spectroscopy (Academic, 1979).

7. Kimura, K., Katsumata, S., Achiba, Y., Yamazaki, T. \& Iwata, S. Handbook of HeI Photoelectron Spectra (Japan Scientific Societies Press, 1981).

8. Becker, U. \& Shirley, D. A. (eds) VUV and Soft X-Ray Photoionization (Plenum Press, 1996).

9. Torres, R. et al. Probing orbital structure of polyatomic molecules by high-order harmonic generation. Phys. Rev. Lett. 98, 203007 (2007).

10. Baker, S. et al. Probing proton dynamics in molecules on an attosecond timescale. Science 312, 424-427 (2006).

11. Smirnova, O. et al. High harmonic interferometry of multi-electron dynamics in molecules. Nature 460, 972-977 (2009).

12. Wörner, H. J., Bertrand, J. B., Kartashov, D. V., Corkum, P. B. \& Villeneuve, D. M. Following a chemical reaction using high-harmonic spectroscopy. Nature 466, 604-607 (2010).

13. Corkum, P. B. Plasma perspective on strong field multiphoton ionization. Phys. Rev. Lett. 71, 1994-1997 (1993).

14. Le, A-T., Lucchese, R. R., Lee, M. T. \& Lin, C. D. Probing molecular frame photoionization via laser generated high-order harmonics from aligned molecules. Phys. Rev. Lett. 102, 203001 (2009).

15. Frolov, M. V. et al. Analytic description of the high-energy plateau in harmonic generation by atoms: Can the harmonic power increase with increasing laser wavelengths? Phys. Rev. Lett. 102, 243901 (2009).

16. Ganeev, R. A. High-order harmonic generation in a laser plasma: A review of recent achievements. J. Phys. B 40, R213-R253 (2007).

17. Frolov, M. V., Manakov, N. L. \& Starace, A. F. Potential barrier effects in high-order harmonic generation by transition-metal ions. Phys. Rev. A 82, 023424 (2010).

18. Schmidt, B. E. et al. Compression of $1.8 \mu \mathrm{m}$ laser pulses to sub two optical cycles with bulk material. Appl. Phys. Lett. 96, 121109 (2010).

19. Frolov, M. V., Manakov, N. L., Sarantseva, T. S. \& Starace, A. F. Analytic formulae for high harmonic generation. J. Phys. B 42, 035601 (2009).

20. Landau, L. D. \& Lifshitz, E. M. Quantum Mechanics Non-Relativistic Theory 3 edn (Course of Theoretical Physics, vol. 3, Pergamon Press, 1977).

21. Huang, K. N., Johnson, W. R. \& Cheng, K. T. Theoretical photoionization parameters for the noble gases argon, krypton, and xenon. At. Nucl. Data Tables 26, 33-45 (1981).

22. Minemoto, S. et al. Retrieving photorecombination cross sections of atoms from high-order harmonic spectra. Phys. Rev. A 78, 061402(R) (2008).

23. Amusia, M. Y. \& Connerade, J-P. The theory of collective motion probed by light. Rep. Prog. Phys. 63, 41-70 (2000).

24. Becker, U. et al. Subshell photoionization of Xe between 40 and $1,000 \mathrm{eV}$. Phys. Rev. A 39, 3902-3911 (1989).

25. Kutzner, M., Radojević, V. \& Kelly, H. P. Extended photoionization calculations for xenon. Phys. Rev. A 40, 5052-5057 (1989).

26. Popmintchev, T. et al. Phase matching of high harmonic generation in the soft and hard X-ray regions of the spectrum. Proc. Natl Acad. Sci. USA 106, 10516-10521 (2009).

27. Vozzi, C. et al. High-order harmonics generated by $1.5 \mu \mathrm{m}$ parametric source. J. Mod. Opt. 57, 1008-1013 (2010).

28. Schultze, M. et al. Delay in photoemission. Science 328, 1658-1662 (2010).

29. Shiner, A. D. et al. Wavelength scaling of high harmonic generation efficiency. Phys. Rev. Lett. 103, 073902 (2009).

30. Farrell, J. P., McFarland, B. K., Bucksbaum, P. H. \& Gühr, M. Calibration of a high harmonic spectrometer by laser induced plasma emission. Opt. Express $17,15134-15144$ (2009).

31. Fahlman, A., Krause, M. O., Carlson, T. A. \& Svensson, A. Xe $5 s, 5 p$ correlation satellites in the region of strong interchannel interactions, $28-75 \mathrm{eV}$. Phys. Rev. A 30, 812-819 (1984).

\section{Acknowledgements}

The authors thank laser technicians F. Poitras and A. Laramée for their dedicated work on the laser system, and gratefully acknowledge financial support from NSERC, AFOSR, CIPI and FQRNT. We thank U. Becker, M. Spanner and T. Starace for illuminating discussions.

\section{Author contributions}

A.D.S. and B.E.S. contributed equally to this work. B.E.S., F.L. and J-C.K. developed the laser source. A.D.S., B.E.S. and C.T.H. carried out the experiments. H.J.W. interpreted the xenon results. A.D.S. analysed the data. S.P. and D.M.V. provided the theoretical parts. A.D.S., H.J.W., P.B.C. and D.M.V. wrote the manuscript.

\section{Additional information}

The authors declare no competing financial interests. Supplementary information accompanies this paper on www.nature.com/naturephysics. Reprints and permissions information is available online at http://npg.nature.com/reprintsandpermissions. Correspondence and requests for materials should be addressed to D.M.V. 